\title{
Validación de la escala barrat de impulsividad (BIS-11) en población bogotana*
}

\author{
Validation of the barrat impulsivity scale (BIS-11) \\ in bogotana population
}

\author{
Sandra Carolina Urrego Barbosa \\ Olga Lucía Valencia Casallas** \\ Javier Villalba \\ Fundación Universitaria Konrad Lorenz \\ Recibido: 26 de enero de 2017 \\ Revisado: 2 de marzo de 2017 \\ Aceptado: 13 de mayo de 2017
}

\section{Resumen}

El presente trabajo se realizó con el fin de validar la Escala de Impulsividad de Barratt (BIs-11) en población bogotana, y de esta forma aportar evidencia empírica que permita unificar los criterios de interpretación y análisis. Participaron 313 personas: 98 privadas de la libertad transitoriamente en Unidad de Reacción Inmediata (URI); 86 diagnosticadas con trastornos relacionados con impulsividad y 126 estudiantes universitarios. Se realizó la adaptación cultural de la prueba por medio de revisión por lingüista, juicio de expertos y pilotaje. Posteriormente, se definió el instrumento sometido a validación. Los resultados arrojaron una fiabilidad de la escala total de a 0,7 y para las sub escalas motora a 0,6 , atencional a 0,5 y no planeada a 0,4 ; el análisis factorial exploratorio identificó 2 factores que explican el 29,77\% de la varianza total. La evidencia permite concluir una calidad psicométrica moderada, por lo cual se recomienda no usar esta versión para diagnóstico y realizar la traducción de la última actualización de la escala original

Palabras clave: conducta impulsiva, adaptación y validación de escalas.

* $\quad$ Artículo de investigación. DOI: http://dx.doi.org/10.15332/s1794-9998.2017.0002.01

** Correspondencia: Olga Lucía Valencia Casallas, Fundación Universitaria Konrad Lorenz. Correo electrónico: olgal.valenciac@konradlorenz.edu.co. Dirección postal: Cra. 9 Bis \#62-43, Bogotá, Colombia 


\section{Abstract}

The present work was carried out in order to validate the Barratt Impulsivity Scale (BIS-11) in the population of Bogota, and thus provide empirical evidence to unify the interpretation and analysis criteria. Participated 313 people: 98 deprived of freedom temporarily in the Unit of Immediate Reaction (URI); 86 diagnosed with disorders related to impulsivity and 126 university students. The cultural adaptation of the test was made through linguistic revision, expert judgment and piloting. Subsequently, the instrument was validated. The results showed a reliability of the total scale of a 0.7 and for motor subscales a 0.6 , attentional a 0.5 and unplanned a 0.4 ; the exploratory factor analysis identified 2 factors that explain $29.77 \%$ of the total variance. The evidence allows to conclude a psychometric quality moderate, for which it is recommended not to use this version for diagnosis and perform the translation of the last update of the original scale

Keywords: impulsive conduct, adaptation and validation of scales.

La impulsividad es un constructo complejo que se ha definido de muchas formas entre ellas Moeller et al. (2001), la conceptualizan como una predisposición a realizar acciones rápidas y no reflexivas en respuesta a estímulos internos y/o externos, a pesar de las consecuencias negativas que podrían tener éstas, tanto para la misma persona, como para terceros. Este constructo ha sido contemplado como un síntoma de algunos trastornos mentales incluyendo déficit de atención / hiperactividad, trastornos bipolar, antisocial y límite de la personalidad y comportamientos suicidas (American Psychiatric Association, 2013). La Escala Barratt de impulsividad cuenta con un desarrollo de más de cinco décadas ( Barrat, 1959, 1965, 1985, 1994) actualmente se encuentra en su versión 11 (BIS-11) la cual se ha convertido en patrón oro para medir la impulsividad, tanto en el campo de la psicología clínica como de la psicología jurídica, al ser usada para correlacionar la impulsividad con trastornos alimenticios (Higgins, Lin, Álvarez, y BardoneCone, 2015; Leitch, Morgan y Yeomans, 2013), síntomas depresivos (Dvorak, Lamis, y Malone, 2013; Jakubczyk, Klimkiewicz, Topolewska-Wochowska, Serafin, Sadowska-Mazuryk \& Pupek-Pyziot, 2012), abuso de sustancias (Dvorak et al., 2013; Heinz, Bui, Thomas, y Blonigen, 2015; Huang, Li, Fang, Wu, y Liao, 2013; Jakubczyk, et al., 2012; Reid, Cyders, Moghaddam, y Fong, 2014), trastorno bipolar (Etain, Mathieu, Liquet, Raust, Cochet \& Richard, 2013), trastorno límite de la personalidad (Van Zutphen, Siep, Jacob, Goebel \& Arntz, 2015), fumadores habituales (Rass, Ahn, y O'Donnell, s.f.), hipersexualidad (Reid et al., 2014), y comportamientos asociados con infracción de normas de tránsito y accidentes automovilísticos (Bıçaksız y Özkan, s.f.), entre otros. También es usada como evidencia en múltiples pericias forenses.

Sin embargo, y pese a su amplia utilización, la investigación psicométrica referente a la estructura interna de la prueba traducida al español ha sido escasa, lo que ha llevado a que en los últimos años se realicen estudios con el objetivo de evidenciar su comportamiento psicométrico (Folino, Escobar-Cordoba y Castillo, 2006; Loyola, 2011; Malloy-Diniz, De Paula, Vasconcelos, De Almondes, Pessoa, Faria, 2015; Oquendo, Baca-Garcia, Graver, Morales, Montalban y Mann, 2001; Reise, Moore, Sabb, Brown y London, 2013; y Stanford, Mathias, Dougherty, Anderson, Patton y Lake (2009). Estos estudios han sido realizados con poblaciones limitadas en Latinoamérica y no se encuentra evidencia empírica del comportamiento de la prueba en población adulta colombiana, pese a su amplio uso en la práctica clínica y forense.

Es importante tener en cuenta que las escalas pueden verse afectadas por la cultura, Muñiz et al. (2013) ponen de manifiesto el peligro de asumir sin más la universalidad de los constructos entre culturas, y aconsejan evaluar el grado o nivel de solapamiento deseado en el constructo, entre la población origen y en la de equivalencia, es por ello que se hace muy importante realizar la adaptación previa a la validación de la BIS-11.

Los resultados de las diferentes validaciones aunque identifican que la escala mide el constructo, evidencian también algunas limitaciones (ver tabla 1). 
Tabla 1.

Resultados de validaciones de la escala BIS 11 español

\begin{tabular}{|c|c|c|c|c|}
\hline Autor & Oquendo y cols & Folino y cols & Loyola & Salvo y Castro \\
\hline Año & 2001 & 2006 & 2011 & 2013 \\
\hline $\mathrm{N}$ & 29 & 180 & 216 & 763 \\
\hline Población & $\begin{array}{l}\text { Pacientes psiquiátricos } \\
\text { ambulatorios bilingües }\end{array}$ & Hombres en cárcel & Mujeres en cárcel & Adolescentes \\
\hline País & $\begin{array}{l}\text { República dominicana, } \\
\text { puerto rico, cuba }\end{array}$ & Argentina & Perú (Lima) & Chile \\
\hline Media escala total & 1,26 & 63 & - & - \\
\hline Alfa Cronbach Total & - & 0,68 & - & 0,77 \\
\hline Motora & - & 0,7 & 0,76 & - \\
\hline No prevista & - & 0,48 & 0,69 & - \\
\hline Cognitiva & - & 0,24 & - & - \\
\hline Calificación & $0,1,3,4$ & $1,2,3,4$ & $1,2,3,4$ & $0,1,3,4$ \\
\hline
\end{tabular}

Nota: Los datos señalados con (-) no son reportados por el autor.

Estas validaciones se han realizado en poblaciones específicas y como lo señala Loyola (2011), existen dificultades con los ítems 4, 15, 20, 23, 27, 30; los cuales no aplican para la población con mucho tiempo en cárcel y penas muy largas. Los estudios presentan diferencias en las escalas Likert bajo las cuales se califica la prueba, y la fiabilidad obtenida por el alfa de Cronbach no es reportada para todas las sub escalas, excepto por Folino et al. (2006), sin embargo, entre los resultados reportados para este coeficiente, se observan diferencias importantes entre los estudios.

Tabla 2.

Resultados de validaciones de la escala BIS 11 en inglés y portugués

\begin{tabular}{|c|c|c|c|}
\hline Autor & Patton et al. & Reise et al. & Malloy-Diniz et al. \\
\hline Año & 1995 & 2013 & 2015 \\
\hline$N$ & 733 & 691 & 3053 \\
\hline Población & $\begin{array}{l}\text { Estudiantes universitarios. } \\
\text { Población psiquiátrica y } \\
\text { privados de la libertad }\end{array}$ & Adultos sanos & Adultos sanos \\
\hline País & Canadá & Estados Unidos & Brasil \\
\hline Media escala total & - & 59.18 & - \\
\hline Alfa Cronbach Total & - & .80 & .79 \\
\hline Motora & - & .70 & .59 \\
\hline No planeada & - & .60 & .59 \\
\hline Atencional & - & .67 & .65 \\
\hline Calificación & $1,2,3,4$ & - & - \\
\hline
\end{tabular}

Nota: Los datos señalados con (-) no son reportados por el autor.
De la misma forma se han encontrado dificultades en la escala en sus versiones en inglés y portugués (Malloy-Diniz et al. 2015; Patton et al., 1995; Reise et al., 2013). Estudios en los que se plantea que pese a las bondades de la prueba en los diferentes ámbitos y al medir la impulsividad en múltiples dimensiones, se hace evidente la necesidad de revisión de los factores, pues estadísticamente se encuentran dos y no tres factores como se planteó inicialmente.

Nota: Los datos señalados con (-) no son reportados por el autor. 
Por lo tanto es pertinente realizar la adaptación cultural del instrumento a la población bogotana, determinar el tipo de calificación más adecuado para su uso, y explorar la fiabilidad y validez de la prueba y sus correspondientes subpruebas.

\section{Metodología}

\section{Participantes}

Participaron 313 sujetos seleccionados a conveniencia a partir de los siguientes criterios: personas mayores de edad y menores de 75 años (teniendo en cuenta las edades usadas en validaciones en Chile y Argentina), que supieran leer y escribir, no se encontraran en estado alterado de conciencia y que pertenecieran a una de estas 3 poblaciones: personas privadas de la libertad en Unidad de Reacción Inmediata de la ciudad de Bogotá (URI) en la localidad de Puente Aranda (presunción de delitos contra la vida y la integridad personal, lesiones personales y actos sexuales abusivos); en tratamiento en clínicas de recuperación mental (Clínica La Inmaculada y Clínica Retornar), con diagnósticos relacionados con impulsividad (trastorno límite de la personalidad, trastorno bipolar, trastorno por control de impulsos, trastorno por comportamientos antisociales y trastorno por dependencia a sustancias) y población universitaria (Fundación Universitaria Konrad Lorenz). Se tuvo en cuenta que ninguna población superara el $70 \%$ del total de la muestra. Se excluyeron sujetos con estado de conciencia alterado y analfabetas.

Se eliminaron 3 sujetos por presentar características de dos de las poblaciones simultáneamente, por lo cual la muestra final fue conformada por 310 sujetos: 98 privados de la libertad en URI, 86 en tratamiento en clínicas de salud mental y 126 cursando estudios universitarios; con edades comprendidas entre los 18 a 66 años, lo que permite obtener una media en la edad de la población de 28.9; 147 hombres y 163 mujeres, distribuidos en todos los estratos socioeconómicos: 50 de estrato 1,73 de estrato 2,145 de estrato 3,34 de estrato 4, 3 de estrato 5 y 5 de estrato 6 ; y con diversos niveles de estudio: 25 con primaria, 43 con con secundaria incompleta, 86 bachilleres, 26 técnicos, 32 tecnólogos, 88 universitarios y 9 especialistas.

\section{Diseño}

La investigación está enmarcada en una metodología cuantitativa y se clasifica como un estudio instrumental (Montero y León, 2007).

\section{Instrumentos}

Cuestionario de datos sociodemográficos: mediante la cual se registraron datos como edad, sexo, estrato socioeconómico, escolaridad, lugar donde se aplica la prueba, autoreporte de diagnósticos realizados por psiquiatría y de vinculación en delitos (ver Anexo A). Este cuestionario se acompañó del consentimiento informado.

Escala de Impulsividad de Barratt-11: escala con 30 ítems cada uno con 4 opciones de respuesta tipo Likert, la cual fue autoaplicada con un promedio de duración de 15 minutos para cada sujeto (ver Anexo B).

La escala fue aplicada bajo protocolo propuesto (ver Anexo C).

\section{Procedimiento}

Se realizó una primera fase, en la que se hizo la adaptación de la escala por medio de una revisión del lingüista. en la cual se comparó la escala Barrat Impulsiveness Scale (BIS-11) (versión en inglés) con la Escala Barrat de Impulsividad (BIS-11) (versión en español), con el objetivo de verificar la literalidad del último documento, en términos de coherencia semántica, cohesión sintáctica, mantenimiento de la direccionalidad del sentido semántico e inferencia de la conservación de la validez de constructo. Para lo cual se contó con el análisis de un lingüista experto, master en psicología clínica y de la salud. Posteriormente se realizó una evaluación por jueces del instrumento traducido, para la cual se contó con la participación de 4 jueces expertos, uno en el área clínica, uno en jurídica, un psicómetra y un lingüista, a quienes se les solicitó calificar el instrumento (ver Anexo D), valorando el contenido de los ítems según su pertinencia, claridad y relevancia; bajo la estricta aplicación del protocolo y el posterior análisis de concordancia, se tuvo en cuenta los porcentajes netos y la W de Kendal. Además se 
realizó un pilotaje, en el que se aplicó la prueba a 15 estudiantes universitarios, 11 mujeres y 4 hombres, estudiantes de postgrado, pertenecientes a estratos 2 al 6 distribuidos así: 2 de estrato 2, 10 de estrato 3, 1 de estrato 4, 1 de estrato 6 y 1 sin dato, con autoreporte negativo frente a presencia de trastornos mentales o haber cometido algún delito. Se realizaron análisis de correlación ítem test (correlacionando puntuación total y subpruebas) y análisis de porcentaje frente al enunciado, ante el cual consideraba era más fácil la respuesta.

Los resultados del análisis lingüístico, la validación de jueces y el pilotaje, analizados en conjunto permitieron obtener el formato definitivo de la prueba para su aplicación (ver Anexo E).

Una segunda fase constituyó la validación en la cual se realizó el procesamiento y análisis de resultados, la determinación formato Likert más preciso para la calificación de la prueba y los análisis confiabilidad, correlación ítem-test, y análisis factorial exploratorio.

\section{Resultados}

Mediante la adaptación y revisión del lingüista se encontró que la versión en español "conserva parcialmente el sentido discursivo de la construcción textual en tanto cohesión y coherencia global, micro y macroestructuctura semántica y superestructura esquemática con respecto de la escala en inglés" (Escobar, 2016, p.). Entre las diferencias encontradas se evidenció cambio en la posición de 17 ítems; adición de sintagma explicativo en los ítems 4, 7, 11,16, 19, 24, 25, 27,29 y 30; alteración total de la literalidad en los ítems 3, 9, 17, 22, 23 y 24, y alteración parcial de la literalidad en el ítem 30. Por lo cual la traducción realizada por Oquendo et al. (2001), no concuerda de forma exacta con la prueba original en inglés.

La evaluación por jueces del instrumento traducido, arrojó un porcentaje de acuerdo neto para claridad del $80 \%$, para pertinencia del $53 \%$ y para relevancia del $36 \%$. Por medio del coeficiente de concordancia de Kendal (W) se obtuvo una concordancia para claridad del 0,098, con una significancia de 0,032; para pertinencia la concordancia encontrada es de
0,079 con una significancia de 0,69 ; y para relevancia la concordancia es de 0,115 con una significancia de 0,016 . Es importante anotar que todos los ítems en los que se encuentran dificultades de parte de los jueces tienen adición de sintagma explicativo, lo cual genera dificultades para su interpretación.

En el pilotaje se encontró una baja correlación ítemtest, tanto con el total, como con la sub escala, de los ítems 13, 14, 17 y 20; y baja correlación con el total, pero no con la sub escala de los ítems $1,8 \mathrm{y}$ 28. Para los enunciados que presentaron adición de sintagma explicativo se solicitó a los participantes que señalaran el sintagma al cual les era más fácil responder, dichas respuestas fueron ponderadas y con base en el porcentaje de escogencia, sugerencia de los jueces y similitud con el enunciado en inglés, se realizó la escogencia del enunciado para el ítem. El ítem 17 fue reemplazado por "me gustan los rompecabezas" ya que presentó muy baja correlación ítem-test, tanto con el total como con la sub escala, los jueces advirtieron de las dificultades contextuales que este ítem podría presentar y no aparece en la versión actual de la prueba en inglés.

En la fase de validación se pudo determinar que la escala de calificación más adecuada para usar es la escala 1, 2, 3, 4, siendo esta, entre las usadas comúnmente, la que menos varianza no explicada, aporta al instrumento y a partir de allí se realizaron los análisis a la prueba.

El análisis de fiabilidad de la prueba arrojó los datos presentados en la tabla 3.

Tabla 3

Fiabilidad para la escala y sub escalas.

\begin{tabular}{lcc}
\hline & Alfa de Cronbach & N de elementos \\
\hline Total & .75 & 30 \\
\hline No planeada & .54 & 12 \\
\hline Motora & .69 & 10 \\
\hline Atencional & .40 & 8 \\
\hline
\end{tabular}

Frente a la correlación ítem-test se evidencian dificultades en 4 de los ítems en la correlación con la escala total, sin embargo, su correlación con la sub escala es superior a 0,25 , excepto para el ítem 30 que tiene dificultades de correlación, tanto con la escala total como con la sub escala a la cual pertenece. 
El Análisis factorial exploratorio sugiere la presencia de 6 factores que explican el 47,20\% de la varianza de la prueba (ver tabla 4). Sin embargo, existen 2 factores a los que se les puede atribuir 29 de los 30 ítems y el 29,77 \% de la varianza explicada. El ítem 30 no presenta un peso relevante para ninguno de los 2 factores evidenciados.

Tabla 4.

Matriz de componentes. Análisis Factorial Exploratorio.

\begin{tabular}{|c|c|c|}
\hline & 1 & 2 \\
\hline ITEM01 & & 0,543 \\
\hline ITEM02 & 0,499 & \\
\hline ITEM03 & 0,359 & \\
\hline ITEM04 & 0,464 & \\
\hline ITEM05 & & 0,59 \\
\hline ITEM06 & & 0,669 \\
\hline ITEM07 & & 0,597 \\
\hline ITEM08 & & 0,53 \\
\hline ITEM09 & 0,506 & \\
\hline ITEM10 & & 0,649 \\
\hline ITEM11 & & 0,50 \\
\hline ITEM12 & 0,488 & \\
\hline ITEM13 & & 0,367 \\
\hline ITEM14 & 0,483 & \\
\hline ITEM15 & 0,604 & \\
\hline ITEM16 & 0,581 & \\
\hline ITEM17 & & 0,469 \\
\hline ITEM18 & 0,572 & \\
\hline ITEM19 & & 0,487 \\
\hline ITEM20 & 0,394 & \\
\hline ITEM21 & 0,458 & \\
\hline ITEM22 & & 0,603 \\
\hline ITEM23 & 0,462 & \\
\hline ITEM24 & 0,502 & \\
\hline ITEM25 & 0,515 & 0,311 \\
\hline ITEM26 & 0,48 & \\
\hline ITEM27 & 0,634 & \\
\hline ITEM28 & 0,382 & \\
\hline ITEM29 & 0,54 & \\
\hline ITEM30 & & 0,153 \\
\hline
\end{tabular}

\section{Discusión}

La revisión del lingüista favoreció la identificación de falencias en la estructura general de la prueba en español que no presenta la prueba actual en inglés, como sintagmas explicativos que podrían llegar a generar diferencias en la interpretación del ítem por parte del sujeto. Stanford et al. (2009) advierte que se han realizado estudios con la BIS-11a, la cual corresponde a una versión de prueba de la BIS-11, y que no se recomienda su uso por tener coincidencia en solo 24 de los ítems, por lo cual se hace evidente que esta fue la prueba usada por Oquendo (2001) para la traducción, sin embargo, esta prueba ha sido ampliamente usada en el contexto de los países de habla hispana, y hasta finales de 2015, cuando se planteó este estudio, esta traducción era aceptada para su uso mediante la página oficial del Dr Stanford".

Dadas las dificultades encontradas desde la traducción de la prueba al español, ya que es coincidente con la BIS-11a que es una versión previa a la BIS-11, no se consideró su traducción completa pues las validaciones realizadas en otros idiomas (Stanford et al., 2009; Malloy-Diniz et al., 2015; y Reise et al.,2013), que incluyen el idioma original de la prueba, muestran las mismas dificultades en cuanto a fiabilidad y claridad en el constructo que las validaciones realizadas con la prueba traducida por Oquendo (2001) que está siendo usada en Colombia y Latinoamérica en práctica clínica y forense, lo cual hace relevante realizar estudios sobre esta versión.

Es importante señalar que una de las principales dificultades en los datos estadísticos de la prueba, es generada por el cambio en la escala y que un gran número de pruebas en la práctica clínica y forense han sido aplicadas bajo la escala 0-1-3-4, la cual genera dificultades importantes, pues como se pudo demostrar, este tipo de calificación incrementa la varianza no explicada por el instrumento.

La fiabilidad total de la prueba y de la escala no planeada son aceptables, sin embargo, no es este el único elemento que debe tenerse en cuenta, es importante evidenciar que solo un ítem presenta

$1 \quad$ www.impulsivity.org 
baja correlación con la sub prueba a la que pertenece y solo cuatro de ellos presentan una baja correlación con la escala total, entre ellos es importante revisar el ítem 30, el cual se usó en la adaptación realizada, se sugiere modificar el sintagma explicativo adicionado por el enunciado inicial, el cual conserva la literalidad del enunciado en inglés.

El análisis factorial sugiere la relevancia de dos de los factores (motor y no planeado) y ponen de nuevo de manifiesto la dificultad de la escala atencional como determinante en el análisis de los resultados, lo cual concuerda con los demás estudios realizados (Folino et al., 2006; Loyola, 2011; MalloyDiniz et al., 2015; Reise et al., 2013).

La ANOVA evidencia el comportamiento de la prueba entre poblaciones, sugirie diferencias significativas en la impulsividad motora para la población carcelaria y en impulsividad no planeada para población psiquiátrica, ello concuerda con las definiciones generadas para cada subprueba (Stanford, 2009).

\section{Conclusiones y recomendaciones}

Es importante tener en cuenta que las escalas son aproximaciones a características no observables directamente (Gómez y Sánchez, 1998), por lo tanto no pueden constituirse en un único elemento diagnóstico, y su validez debe ser construida por medio de su aplicación y análisis (Gómez y Sánchez, 1998; Gómez e Hidalgo, 2009). Por ello el presente estudio se aproxima a una interpretación adecuada de la escala BIS-11, sugiere usar la calificación 1, 2, 3,4 y tener en cuenta para su análisis las calificaciones por sub escalas, no únicamente por el total.

Se recomienda para futuras aplicaciones la modificación del ítem 30 y utilizar la traducción del ítem usada en la versión en inglés. También se considera importante realizar una aproximación a las diferencias en fiabilidad, correlaciones y comportamiento interpoblacional a la escala traducida de la original y compararla con los resultados obtenidos en este estudio, esto con el fin de poner en evidencia si las diferencias entre las dos escalas son causantes de las dificultades encontradas.

\section{Referencias}

American Psychological Association (APA), American Educational Research Association (AERA), y National Council on Measurement in Education (NCME). (2014). Technical recommendations for psychological test and diagnostic techniques. Washington D.C: American Psychological Association.

Barratt, E.S. (1959). Anxiety and impulsiveness Related to Psychomotor Efficiency. Perceptual and Motor Skills. Southern Universities Press. 191-198.

Barratt, E.S. (1965). Factor Analysis of Some Psychometric Measures of Impulsiveness and Anxiety. Psychological Reports, (xx)xx . 547-554.

Barratt, E. S. (1985). Impulsiveness subtraits: Arousal and information processing. In J.T. Spence \& C.E. Izard (Eds.), Motivation, emotion and personality.(pp. 137-146). North Holland: Elsevier Science Publishers.

Barratt, E. S. (1994). Impulsiveness and aggression. In J. Monahan y H.J. Steadman (Eds.), Violence and mental disorder: Developments in risk as sessment (pp. 61-79). Chicago, IL: University of Chicago Press.

Bıçaksız, P., \& Özkan, T. (SF). Impulsivity and driver behaviors, offences and accident involvement: A systematic review. Transportation Research Part F: Traffic Psychology and Behaviour. doi:http://dx.doi.org/10.1016/j.trf.2015.06.001

Dvorak, R.D., Lamis, D.A., \& Malone, P.S. (2013). Alcohol use, depressive symptoms, and impulsivity as risk factors for suicide proneness among college students. Journal of Affective Disorders, 149, 326-334. doi:http://dx.doi. org/10.1016/j.jad.2013.01.046

Escobar, F. (2016). Escala Barrat de Impulsividad (BIS-11). Un análisis de la versión traducida al español. Manuscrito inédito. Bogotá.

Etain, B., Mathieu, F., Liquet, S., Raust, A., Cochet, B., Richard, J.R. y cols. (2013). Clinical features associated with trait-impulsiveness in 
euthymic bipolar disorder patients. Journal of Affective Disorders, 144, 240-247. doi:http:// dx.doi.org/10.1016/j.jad.2012.07.005

Folino, J. Escobar-Cordoba, F. y Castillo, J. (2006). Exploración de la validez de la escala de impulsividad de Barratt (BIS 11) en la población carcelaria Argentina. Revista Colombiana de Psiquiatría, XXXV(2), 132-148.

Gómez, J. e Hidalgo, M. (2009). La validez en los tests, escalas y cuestionarios. Colombia: Centro de estudios de opinión.

Gómez, C. y Sánchez, R. (1998) Conceptos básicos sobre validación de escalas. Revista Colombiana de Psiquiatría, XXVII.

Heinz, A.J., Bui, L., Thomas, K.M., \& Blonigen, D.M. (2015). Distinct facets of impulsivity exhibit differential associations with substance use disorder treatment processes: A cross-sectional and prospective investigation among military veterans. Journal of Substance Abuse Treatment, 55, 21-28. doi:http://dx.doi. org/10.1016/j.jsat.2015.02.005

Higgins, M.K., Lin, S.L., Alvarez, A., \& Bardone-Cone, A.M. (2015). Examining impulsivity as a moderator of the relationship between body shame and bulimic symptoms in Black and White young women. Body Imagin, 14, 39-46. doi:http:// dx.doi.org/10.1016/j.bodyim.2015.03.007

Huang, C.Y., Li, C.S.R., Fang, S.C., Wu, C.S., y Liao, D.L. (2013). The reliability of the Chinese version of the Barratt Impulsiveness Scale version 11 , in abstinent, opioid-dependent participants in Taiwan. Journal of the Chinese Medical Association, 76, 289-295. doi:http:// dx.doi.org/10.1016/j.jcma.2013.01.005

Jakubczyk, A., Klimkiewicz, A., TopolewskaWochowska, A., Serafin, P., Sadowska-Mazuryk, J., Pupek-Pyziot, J. y cols (2012). Relationships of impulsiveness and depressive symptoms in alcohol dependence. Journal of Affective Disorders, 136. 841-847. doi:http://dx.doi. org/10.1016/j.jad.2011.09.028
Leitch, M., Morgan, M., y Yeomans, M. (2013). Different subtypes of impulsivity differentiate uncontrolled eating and dietary restraint. Appetite, 69, 54-63. doi:http://dx.doi. org/10.1016/j.appet.2013.05.007

Loyola, N. (2011). Validez y confiabilidad de la escala de impulsividad de Barratt versión 11 (BIS-11) en mujeres encarceladas. (Tesis doctoral licenciatura). Pontificia Universidad Católica Perú.

Malloy-Diniz, L., De Paula, J.; Vasconcelos, J., De Almondes, K., Pessoa, R., Faria,L. et al. (2015). Normative data of the Barratt Impulsiveness Scale 11 (BIS-11) for Brazilian adults. Revista Brasileira de Psiquiatria, 37, 245-248.

Moeller, Barratt, Dougherty, Schmitz, \& Swann, (2001). Psiquiatric aspects of impulsivity. The American Journal of Psiquiatric, 158, 1783-93.

Montero , I, León O (2007) A guide for naming research studies in Psychology. International Journal of Clinical and Health Psychology. 7 (3) $847-862$

Muñiz, J., Elosua, P. y Hambleton, R. (2013). Directrices para la traducción y adaptación de los tests: segunda edición. Psicothema, 25, 151-157.

Oquendo, M.A., Baca-García, E., Graver, R., Morales, M., Montalban, V., y Mann, J. J. (2001). Spanish adaption of the Barratt impulsiveness scale (BIS). European Journal of Psychiatry. 15, 147-155.

Patton, J.H., Stanford, M.S. \& Barratt, E.S. (1995). Factor structure of the Barratt Impulsiveness Scale. Journal of Clinical Psychology, 51, 768-774. doi:10.1002/1097-4679(199511)51:6<768::aidjclp2270510607>3.0.CO;2-1.

Rass, O., Ahn, W.Y., y O'Donnell, B.F. (s.f.). Resting-state EEG, impulsiveness, and personality in daily and nondaily smokers. Clinical Neurophysiology. doi:http://dx.doi. org/10.1016/j.clinph.2015.05.007

Reid, R.C., Cyders, M.A., Moghaddam, J.F., y Fong, T.W. (2014). Psychometric properties 
of the Barratt Impulsiveness Scale in patients with gambling disorders, hypersexuality, and methamphetamine dependence. Addictive Behaviors, 39, 1640-1645. doi:http://dx.doi. org/10.1016/j.addbeh.2013.11.008

Reise, S., Moore,T., Sabb, F., Brown, A., y London, E. (2013). The Barratt Impulsiveness Scale-11: Reassessment of its Structure in a community sample. Psychological Assessment, 25, 631-642.

Salvo G, Lilian, \& Castro S, Andrea. (2013). Confiabilidad y validez de la escala de impulsividad de Barratt (BIS-11) en adolescentes. Revista chilena de neuro-psiquiatría, 51(4), 245-254. https://dx.doi.org/10.4067/ S0717-92272013000400003
Stanford, M., Mathias, C., Dougherty, D., Anderson, N., Patton, J. y Lake, S. (2009). Fifty years of the Barratt Impulsiveness Scale: An update and review. Personality and Individual Differences, 47, 385-395.

Van Zutphen, L., Siep, N., Jacob, G.A., Goebel, R., y Arntz, A. (2015). Emotional sensitivity, emotion regulation and impulsivity in borderline personality disorder: A critical review of $\mathrm{fMRI}$ studies. Neuroscience \& Biobehavioral Reviews. 51, 64-76. doi: http://dx.doi.org/10.1016/j. neubiorev.2015.01.001 


\section{Anexo A}

\section{VALIDACIÓN DE LA ESCALA BARRAT DE IMPULSIVIDAD (BIS-11) EN POBLACIÓN BOGOTANA.}

El presente trabajo se realiza con el fin de validar la Escala de Impulsividad de Barrat (BIS-11) en población Bogotana, y de esta forma aportar evidencia empírica que permita unificar los criterios de interpretación y análisis de la escala en ésta población. Por ello le solicitamos diligenciar la escala con la mayor sinceridad posible, los resultados de esta aplicación pretenden evaluar el contenido de la prueba en relación a la medición de la impulsividad, lo cual corresponde a un procedimiento estadístico que se obtiene como resultado del análisis de las respuestas de los 300 sujetos a los que se les aplicará, resultados que serán entregados a la institución, sin realizar valoraciones individuales.

Esta investigación se realiza teniendo en cuenta la Ley 1090 del 2006, en el Título II, Articulo 8 se menciona que evitarán el uso indebido de los resultados de la evaluación. Respetarán el derecho de los usuarios de conocer los resultados. Artículo 9, menciona sobre la Investigación con participantes humanos, que el psicólogo la aborda respetando la dignidad y el bienestar de las personas que participan, con pleno conocimiento de las normas legales. En el Artículo 47: “El psicólogo tendrá el cuidado necesario en la presentación de resultados, hasta tanto estén debidamente validadas y estandarizadas.
Una vez informado sobre los procedimientos que se llevarán a cabo, la importancia de los mismos para la información, Acepto , No Acepto . Con respecto a mis resultados individuales Deseo No Deseo recibir retroalimentación. Si su respuesta es afirmativa coloque acá su correo electrónico:

Firma

\section{Por favor diligencie la siguiente información, recuerde que se le dará un uso confidencial:}

Edad: Género: Estrato: Nivel de estudios:

¿Ha sido acusado de algún delito?: ¿Cuál?

¿Ha sido diagnosticado con alguna enfermedad mental?

¿Cuál? 


\section{Anexo B}

\section{Evaluación personal BIS-11:}

Las personas son diferentes en la forma en la que se comportan y piensan en distintas situaciones. Esta es una prueba para medir algunas de las formas en que usted actúa y piensa. No se detenga demasiado tiempo en ninguna de las oraciones. Responda rápida y honestamente.

\section{Raramente o nunca Ocasionalmente A menudo Siempre o casi siempre \\ 1

2

12234

1 Planifico mis tareas con cuidado.

2 Hago las cosas sin pensarlo.

3 Casi nunca me tomo las cosas a pecho. (No me perturbo con Facilidad).

4 Mis pensamientos pueden tener gran velocidad. (Tengo pensamientos

que van muy rápido en mi mente).

5 Planifico mis viajes con antelación.

6 Soy una persona con autocontrol.

7 Me concentro con facilidad. (Se me hace fácil concentrarme).

8 Ahorro con regularidad.

9 Se me hace difícil estar quieto por largos periodos de tiempo.

10 Pienso las cosas cuidadosamente.

11 Planifico para tener un trabajo fijo. (Me esfuerzo por asegurar que tendré dinero

11 para pagar por mis gastos).

12 Digo las cosas sin pensarlas.

13 Me gusta pensar sobre problemas complicados. (Me gusta pensar sobre problemas complejos).

14 Cambio de trabajo frecuentemente. (No me quedo en el mismo trabajo

14 por largos periodos de tiempo).

15 Actúo impulsivamente.

16 Me aburro con facilidad tratando de resolver problemas en mi mente.

16 (Me aburre pensar en algo por demasiado tiempo).

17 Visito al médico y al dentista con regularidad.

18 Hago las cosas en el momento en que se me ocurren.

19 Soy una persona que piensa sin distraerse. (Puedo enfocar mi mente

19 en una sola cosa por mucho tiempo).

20 Cambio de vivienda a menudo. (Me mudo con frecuencia

20 o no me gusta vivir en el mismo sitio por mucho tiempo).

21 Compro cosas impulsivamente.

22 Yo termino lo que empiezo.

23 Camino y me muevo con rapidez.

24 Resuelvo los problemas experimentando. (Resuelvo los problemas

24 tratando una posible solución y viendo si funciona).

25 Gasto efectivo o en crédito más de lo que gano. (Gasto más de lo que gano)

26 Hablo rápido.

27 Tengo pensamientos extraños cuando estoy pensando.

(A veces tengo pensamientos irrelevantes cuando pienso).

28 Me interesa más el presente que el futuro.

29 Me siento inquieto en clases o charlas. (Me siento inquieto si tengo

29 que oír a alguien hablar por un largo periodo de tiempo).

30 Planifico para el futuro. (Me interesa más el futuro que el presente).

Agradecemos sus valiosos aportes a este trabajo. 


\section{Anexo C \\ VALIDACIÓN DE LA ESCALA BARRAT DE IMPULSIVIDAD (BIS-11) EN POBLACIÒN BOGOTANA.}

\section{PROTOCOLO DE APLICACIÓN.}

- La prueba será auto aplicada y su diligenciamiento tarda aproximadamente 10 minutos.

- Solicite a la persona que lea el consentimiento informado y marque si acepta o no según considere y aclare las dudas en caso de que las tenga.

- Tenga en cuenta que hay un apartado donde el participante manifiesta su deseo o no de recibir retroalimentación individual. Verifique que lo diligencie y en caso de que la respuesta sea afirmativa, revise que el correo electrónico este escrito claramente.

- Si la persona no acepta la participación reciba el instrumento; si acepta permítale pasar a la hoja dos.

- A partir de allí, no realice aclaraciones. Si surgen dudas refiérale al sujeto que responda según comprenda.

- Al recoger la escala verifique que la información sociodemográfica sea clara y esté completa.

- Revise que la prueba esta diligenciada completamente.

- Agradezca al sujeto por su participación. 


\section{Anexo D \\ VALIDACIÓN DE LA ESCALA BARRAT DE IMPULSIVIDAD (BIS-11) EN POBLACIÒN BOGOTANA.}

El presente trabajo se realiza con el fin de validar la Escala de Impulsividad de Barrat (BIS-11) en población Bogotana, y de esta forma aportar evidencia empírica que permita unificar los criterios de interpretación y análisis de la escala en ésta población. Como parte de esta validación se realizará la adaptación cultural, para la cual se solicita su participación como juez experto en el área (psicología clínica, psicología jurídica, psicometría), con el fin de obtener un criterio de validez de contenido del instrumento, según su pertinencia, claridad y relevancia.

Para ello se le solicita que califique el ítem de 1 a 3 según el acuerdo al criterio solicitado, de la siguiente manera:

\section{Claridad:}

1. El ítem no es comprensible para la población bogotana.

2. El ítem debe ser ligeramente modificado para ser compresible para la población bogotana

3. El ítem es compresible para la población bogotana.

\section{Pertinencia:}

1. El ítem no corresponde a aspectos relacionados con conductas impulsivas.

2. El ítem debe ser ligeramente modificado para que haga referencia a aspectos relacionados con conductas impulsivas

3. El ítem hace referencia a aspectos relacionados con conductas impulsivas.

\section{Relevancia:}

1. El ítem no es importante para definir aspectos relacionados con conductas impulsivas.

2. El ítem debe ser modificado para considerarse importante para definir aspectos relacionados con conductas impulsivas.

3. El ítem es importante para definir aspectos relacionados con conductas impulsivas.

Para ello se define la impulsividad siguiendo a Moeller et al.(2001), como la predisposición a realizar acciones rápidas y no reflexivas en respuesta a estímulos internos y/o externos, a pesar de las consecuencias negativas que podrían tener éstas, tanto para la misma persona, como para terceros.

Teniendo en cuenta que la escala mide tres subtipos de impulsividad, según Baarrat (1985) estos se definen a continuación seguidos de cada grupo de ítems que hacen referencia al mismo.

Impulsividad atencional: No elaboración de un pensamiento para la acción correspondiente. 
4 Mis pensamientos pueden tener gran velocidad.

4 (Tengo pensamientos que van muy rápido en mi mente).

7 Me concentro con facilidad. (Se me hace fácil concentrarme).

10 Pienso las cosas cuidadosamente.

13 Me gusta pensar sobre problemas complicados.

13 (Me gusta pensar sobre problemas complejos).

16 Me aburro con facilidad tratando de resolver problemas

16 en mi mente. (Me aburre pensar en algo por demasiado tiempo).

19 Soy una persona que piensa sin distraerse.

19 (Puedo enfocar mi mente en una sola cosa por mucho tiempo).

24 Resuelvo los problemas experimentando. (Resuelvo los problemas

24 tratando una posible solución y viendo si funciona).

27 Tengo pensamientos extraños cuando estoy pensando.

27 (A veces tengo pensamientos irrelevantes cuando pienso).

Impulsividad motora: Reacción que tiene el sujeto de manera musculo esquelética

\begin{tabular}{|c|c|c|c|c|}
\hline & Motora & CLARIDAD & PERTINENCIA & RELEVANCIA \\
\hline 2 & Hago las cosas sin pensarlo. & & & \\
\hline 6 & Soy una persona con autocontrol. & & & \\
\hline 9 & Se me hace difícil estar quieto por largos periodos de tiempo. & & & \\
\hline 12 & Digo las cosas sin pensarlas. & & & \\
\hline 15 & Actúo impulsivamente. & & & \\
\hline 18 & Hago las cosas en el momento en que se me ocurren. & & & \\
\hline 21 & Compro cosas impulsivamente. & & & \\
\hline 23 & Camino y me muevo con rapidez. & & & \\
\hline 26 & Hablo rápido. & & & \\
\hline 29 & $\begin{array}{l}\text { Me siento inquieto en clases o charlas. (Me siento inquieto si } \\
\text { tengo que oír a alguien hablar por un largo periodo de tiempo). }\end{array}$ & & & \\
\hline
\end{tabular}

Impulsividad no planeada: Tendencia a no planificar.

\begin{tabular}{|c|c|c|}
\hline & No planeada & PERTINENCIA RELEVANCIA \\
\hline 1 & Planifico mis tareas con cuidado. & \\
\hline 3 & Casi nunca me tomo las cosas a pecho. (No me perturbo con Facilidad). & \\
\hline 5 & Planifico mis viajes con antelación. & \\
\hline 8 & Ahorro con regularidad. & \\
\hline 11 & $\begin{array}{l}\text { Planifico para tener un trabajo fijo. (Me esfuerzo por asegurar que } \\
\text { tendré dinero para pagar por mis gastos). }\end{array}$ & \\
\hline 14 & $\begin{array}{l}\text { Cambio de trabajo frecuentemente. (No me quedo en el mismo traba- } \\
\text { jo por largos periodos de tiempo). }\end{array}$ & \\
\hline 17 & Visito al médico y al dentista con regularidad. & \\
\hline 20 & $\begin{array}{l}\text { Cambio de vivienda a menudo. (Me mudo con frecuencia o no me } \\
\text { gusta vivir en el mismo sitio por mucho tiempo). }\end{array}$ & \\
\hline 22 & Yo termino lo que empiezo. & \\
\hline & $\begin{array}{l}\text { Gasto efectivo o en crédito más de lo que gano. (Gasto más de lo que } \\
\text { gano) }\end{array}$ & \\
\hline 28 & Me interesa más el presente que el futuro. & \\
\hline 30 & Planifico para el futuro. (Me interesa más el futuro que el presente). & \\
\hline
\end{tabular}

Agradecemos sus valiosos aportes a este trabajo. 


\section{Anexo $\mathrm{E}$ ESCALA BIS-11}

Las personas son diferentes en la forma en la que se comportan y piensan en distintas situaciones. Esta es una prueba para medir algunas de las formas en que usted actúa y piensa. No se detenga demasiado tiempo en ninguna de las oraciones. Responda rápida y honestamente, con una $\mathrm{X}$ según la siguiente información:

$\begin{array}{llcc}\text { Raramente o nunca } & \text { Ocasionalmente } & \text { A menudo } & \text { Siempre o casi siempre } \\ 1 & 2 & 3 & 4\end{array}$

$\begin{array}{llll}1 & 2 & 3 & 4\end{array}$

1 Planifico mis tareas con cuidado.

2 Hago las cosas sin pensarlo.

3 Casi nunca me tomo las cosas a pecho.

4 Mis pensamientos pueden tener gran velocidad.

5 Planifico mis viajes con antelación.

6 Soy una persona con autocontrol.

7 Se me hace fácil concentrarme.

8 Ahorro con regularidad.

9 Se me hace difícil estar quieto por largos periodos de tiempo.

10 Pienso las cosas cuidadosamente.

11 Planifico para tener un trabajo fijo.

12 Digo las cosas sin pensarlas.

13 Me gusta pensar sobre problemas complejos.

14 No me quedo en el mismo trabajo por largos periodos de tiempo.

15 Actúo impulsivamente.

16 Me aburro con facilidad tratando de resolver problemas en mi mente.

17 Me gustan los rompecabezas.

18 Hago las cosas en el momento en que se me ocurren.

19 Soy una persona que piensa sin distraerse.

20 No me gusta vivir en el mismo sitio por mucho tiempo.

21 Compro cosas impulsivamente.

22 Yo termino lo que empiezo.

23 Camino y me muevo con rapidez.

24 Resuelvo los problemas experimentando.

25 Gasto más de lo que gano.

26 Hablo rápido.

27 Tengo pensamientos extraños.

28 Me interesa más el presente que el futuro.

29 Me siento inquieto en conferencias.

30 Me interesa más el futuro que el presente. 